JOURNAL OF INTEGRAL EQUATIONS

AND APPLICATIONS

Volume 17, Number 4, Winter 2005

\title{
A CLASS OF MAXIMAL OPERATORS RELATED TO ROUGH SINGULAR INTEGRALS ON PRODUCT SPACES
}

\author{
H. AL-QASSEM AND Y. PAN
}

\begin{abstract}
This paper is concerned with studying the $L^{p}$ boundedness of a class of maximal operators $\mathcal{S}_{\Omega}^{(\gamma)}$ related to rough singular integrals on product spaces. We obtain appropriate $L^{p}$ bounds for such maximal operators and establish the optimality of our condition on the kernel for the $L^{2}$ boundedness of $\mathcal{S}_{\Omega}^{(2)}$. Our results improve substantially the main result obtained by Ding in [8].
\end{abstract}

1. Introduction and statement of results. Throughout this paper, we let $\xi^{\prime}$ denote $\xi /|\xi|$ for $\xi \in \mathbf{R}^{n} \backslash\{0\}$ and $p^{\prime}$ denote the exponent conjugate to $p$, that is, $1 / p+1 / p^{\prime}=1$. Let $n, m \geq 2$. Suppose that $\mathbf{S}^{d-1}$ $(d=n$ or $m)$ is the unit sphere of $\mathbf{R}^{d}$ equipped with the normalized Lebesgue measure $d \sigma=d \sigma\left(x^{\prime}\right)$.

In [7], Chen and Lin studied the $L^{p}$ boundedness of a class of maximal operators $\mathcal{M}_{\Omega}^{(\gamma)}$ defined by

$$
\mathcal{M}_{\Omega}^{(\gamma)} f(x)=\left.\sup _{h}\left|\int_{\mathbf{R}^{n}} f(x-y) h(|y|) \Omega(y /|y|)\right| y\right|^{-n} d y \mid,
$$

where the supremum is taken over the set $\left\{h:\|h\|_{L^{\gamma}\left(\mathbf{R}^{+}, d r / r\right)} \leq 1\right\}$, $\gamma>1$ and $\Omega \in L^{1}\left(\mathbf{S}^{n-1}\right)$ is a function satisfying the cancelation condition

$$
\int_{\mathbf{S}^{n-1}} \Omega\left(y^{\prime}\right) d \sigma\left(y^{\prime}\right)=0 .
$$

Chen and Lin in [7] proved the $L^{p}$ boundedness of the maximal operator $\mathcal{M}_{\Omega}^{(\gamma)}$ under a smoothness condition on $\Omega$ as described in the following theorem:

2000 AMS Mathematics Subject Classification. Primary 42B20, Secondary 42B15, 42B25.

Key words and phrases. Rough kernel, singular integral, product domains.

Received by the editors in December 2004, and in revised form on November 1 , 2005.

Copyright (C)2005 Rocky Mountain Mathematics Consortium 
Theorem A [7]. Assume $n \geq 2$ and $\Omega \in C\left(\mathbf{S}^{n-1}\right)$ satisfying (1.1). Then

$$
\left\|\mathcal{M}_{\Omega}^{(\gamma)}(f)\right\|_{L^{p}\left(\mathbf{R}^{n}\right)} \leq C_{p}\|f\|_{L^{p}\left(\mathbf{R}^{n}\right)}
$$

for $n \gamma /(n \gamma-1)<p<\infty, 1 \leq \gamma \leq 2$, and $f \in L^{p}$. Moreover, the range of $p$ is the best possible.

On the other hand, the corresponding maximal operator of $\mathcal{M}_{\Omega}^{(\gamma)}$ on the product space $\mathbf{R}^{n} \times \mathbf{R}^{m}$ is defined by

$$
\begin{aligned}
& (1.2) \mathcal{S}_{\Omega}^{(\gamma)} f(x, y) \\
& =\left.\sup _{h \in \mathcal{B}_{(\gamma)}}\left|\int_{\mathbf{R}^{n} \times \mathbf{R}^{m}} f(x-u, y-v) h(|u|,|v|) \Omega\left(u^{\prime}, v^{\prime}\right)\right| u\right|^{-n}|v|^{-n} d u d v \mid
\end{aligned}
$$

where $\mathcal{B}^{(\gamma)}$ is the set of all radial functions $h(s, t)$ with

$$
\|h\|_{L^{\gamma}\left(\mathbf{R}^{+} \times \mathbf{R}^{+}, d s d t /(s t)\right)} \leq 1
$$

and $\Omega$ is a function on $\mathbf{R}^{n} \times \mathbf{R}^{m}$ satisfying the following conditions:

$$
\left\{\begin{array}{l}
\int_{\mathbf{S}^{n-1}} \Omega\left(u^{\prime}, \cdot\right) d \sigma\left(u^{\prime}\right)=0, \\
\int_{\mathbf{S}^{m-1}} \Omega\left(\cdot, v^{\prime}\right) d \sigma\left(v^{\prime}\right)=0,
\end{array}\right.
$$

and

$$
\Omega \in L^{1}\left(\mathbf{S}^{n-1} \times \mathbf{S}^{m-1}\right),
$$

$$
\Omega(t x, s y)=\Omega(x, y) \quad \text { for any } \quad t, s>0 .
$$

Recently, Ding in [8] obtained the following $L^{2}$ boundedness of $\mathcal{S}_{\Omega}^{(\gamma)}$ when $\gamma=2$ :

Theorem A. Assume that $n, m \geq 2$ and $\Omega$ satisfies (1.3)-(1.4). Then $\mathcal{S}_{\Omega}^{(2)}$ is bounded on $L^{2}\left(\mathbf{R}^{n} \times \mathbf{R}^{m}\right)$ if $\Omega \in L(\log L)^{2}\left(\mathbf{S}^{n-1} \times \mathbf{S}^{m-1}\right)$. 
Here, a function $\Omega$ belongs to the class $L(\log L)^{\alpha}\left(\mathbf{S}^{n-1} \times \mathbf{S}^{m-1}\right)$ if

$$
\begin{aligned}
& \|\Omega\|_{L(\log L)^{\alpha}\left(\mathbf{S}^{n-1} \times \mathbf{S}^{m-1}\right)} \\
& \quad=\int_{\mathbf{S}^{n-1} \times \mathbf{S}^{m-1}}|\Omega(x, y)| \log ^{\alpha}(2+|\Omega(x, y)|) d \sigma(x) d \sigma(y)<\infty .
\end{aligned}
$$

A question which arises naturally in light of Theorem $\mathrm{A}$ is the following:

Question. Does the $L^{p}$ boundedness of $\mathcal{S}_{\Omega}^{(\gamma)}$ hold for some $p \neq 2$ under a condition in the form $\Omega \in L(\log L)^{\alpha}\left(\mathbf{S}^{n-1} \times \mathbf{S}^{m-1}\right)$, and what is the best possible value of the exponent $\alpha$ so that the $L^{2}$ boundedness of $\mathcal{S}_{\Omega}^{(\gamma)}$ holds.

The main purpose of this paper is to obtain an answer to this question. In fact, we prove the following:

Theorem 1.1. Assume that $n, m \geq 2$ and $\Omega$ satisfies (1.3)-(1.4). Then

(a) If $\Omega \in L(\log L)^{2 / \gamma^{\prime}}\left(\mathbf{S}^{n-1} \times \mathbf{S}^{m-1}\right)$, $S_{\Omega}^{(\gamma)}$ is bounded on $L^{p}\left(\mathbf{R}^{n} \times\right.$ $\left.\mathbf{R}^{m}\right)$ for $\gamma^{\prime} \leq p<\infty$ if $1<\gamma \leq 2$; and it is bounded on $L^{\infty}\left(\mathbf{R}^{n} \times \mathbf{R}^{m}\right)$ if $\gamma=1$;

(b) There exists an $\Omega$ which lies in $L(\log L)^{1-\varepsilon}\left(\mathbf{S}^{n-1} \times \mathbf{S}^{m-1}\right)$ for all $\varepsilon>0$ and satisfies (1.3) such that $S_{\Omega}^{(2)}$ is not bounded on $L^{2}\left(\mathbf{R}^{n} \times \mathbf{R}^{m}\right)$.

We remark that, for any $q>1$, the following inclusions hold and are proper:

$$
C^{1}\left(\mathbf{S}^{n-1} \times \mathbf{S}^{m-1}\right) \subset L^{q}\left(\mathbf{S}^{n-1} \times \mathbf{S}^{m-1}\right) \subset L(\log L)\left(\mathbf{S}^{n-1} \times \mathbf{S}^{m-1}\right),
$$

and

$$
L(\log L)^{\beta}\left(\mathbf{S}^{n-1} \times \mathbf{S}^{m-1}\right) \subset L(\log L)^{\alpha}\left(\mathbf{S}^{n-1} \times \mathbf{S}^{m-1}\right) \quad \text { for } \quad \alpha<\beta .
$$


Clearly, part (a) of Theorem 1.1 represents a substantial improvement in both the range of $p$ and $\Omega$ of the main result of Ding [8], while part (b) shows that the condition $\Omega \in L(\log L)^{2 / \gamma^{\prime}}\left(\mathbf{S}^{n-1} \times \mathbf{S}^{m-1}\right)$ is the best possible in the case $\gamma=2$.

The method employed in this paper allows us to treat a more general class of maximal operators than those given by (1.2). To give a full statement of our results, we let $\Phi$ and $\Psi$ be suitable functions defined on $\mathbf{R}^{+}$. For an $\Omega$ satisfying (1.3)-(1.4), we define the operator $\mathcal{S}_{\Omega, \Phi, \Psi}^{(\gamma)}$ on $\mathbf{R}^{n} \times \mathbf{R}^{m}$ by

$$
\begin{aligned}
\left(\mathcal{S}_{\Omega, \Phi, \Psi}^{(\gamma)} f\right)(x, y)=\sup _{b \in \mathcal{B}} & \mid \int_{\mathbf{R}^{n} \times \mathbf{R}^{m}} f\left(x-\Phi(|u|) u^{\prime}, y-\Phi(|v|) v^{\prime}\right) \\
& \times b(|u|,|v|) \Omega\left(u^{\prime}, v^{\prime}\right)|u|^{-n}|v|^{-n} d u d v \mid .
\end{aligned}
$$

Since $\mathcal{S}_{\Omega, \Phi, \Psi}^{(\gamma)}=\mathcal{S}_{\Omega}^{(\gamma)}$ when $\Phi(t) \equiv \Psi(t) \equiv t$, part (a) of Theorem 1.1 is a special case of the following theorem whose proof will be given in Section 4.

Theorem 1.2. Assume that $n, m \geq 2$ and $\Omega$ satisfies (1.3)-(1.4). Let $S_{\Omega, \Phi, \Psi}^{(\gamma)}$ be given as in (1.5) with $1 \leq \gamma \leq 2$. Assume that $\Phi$ and $\Psi$ are in $C^{2}([0, \infty))$, convex and increasing functions with $\Phi(0)=\Psi(0)=0$.

(a) If $\Omega \in L(\log L)^{2 / \gamma^{\prime}}\left(\mathbf{S}^{n-1} \times \mathbf{S}^{m-1}\right), S_{\Omega, \Phi, \Psi}^{(\gamma)}$ is bounded on $L^{p}\left(\mathbf{R}^{n} \times\right.$ $\mathbf{R}^{m}$ ) for $\gamma^{\prime} \leq p<\infty$ if $1<\gamma \leq 2$; and it is bounded on $L^{\infty}\left(\mathbf{R}^{n} \times \mathbf{R}^{m}\right)$ if $\gamma=1$;

(b) If $\Omega \in L^{q}\left(\mathbf{S}^{n-1} \times \mathbf{S}^{m-1}\right)$, for some $q>1, S_{\Omega, \Phi, \Psi}^{(\gamma)}$ is bounded on $L^{p}\left(\mathbf{R}^{n} \times \mathbf{R}^{m}\right)$ for $\max \left\{\gamma^{\prime} n \delta /\left(\gamma^{\prime} n+n \delta-\gamma^{\prime}\right), \gamma^{\prime} m \delta /\left(\gamma^{\prime} m+m \delta-\gamma^{\prime}\right)\right\}<$ $p<\infty$, where $\delta=\max \left\{2, q^{\prime}\right\}$.

Throughout the rest of the paper the letter $C$ will stand for a constant but not necessarily the same one in each occurrence. 
2. Proof of Theorem 1.1 (b). We follow a similar argument as in [1]. By duality, the operator $\mathcal{S}_{\Omega}^{(2)}$ is simply

$$
\begin{aligned}
\mathcal{S}_{\Omega}^{(2)} f(x, y)=\left(\int_{(0, \infty) \times(0, \infty)} \mid\right. & \int_{\mathbf{S}^{n-1} \times \mathbf{S}^{m-1}} f(x-r \xi, y-t \eta) \\
& \left.\times\left.\Omega(\xi, \eta) d \sigma(\xi) d \sigma(\eta)\right|^{2} \frac{d r d t}{r t}\right)^{1 / 2} .
\end{aligned}
$$

It is obvious that $\mathcal{S}_{\Omega}^{(2)}$ is bounded on $L^{2}\left(\mathbf{R}^{n} \times \mathbf{R}^{m}\right)$ if and only if the multiplier

$$
\begin{aligned}
m(\xi, \eta)=\left(\int_{(0, \infty) \times(0, \infty)} \mid \int_{\mathbf{S}^{n-1} \times \mathbf{S}^{m-1}} e^{-2 \pi i\left(t \xi^{\prime} \cdot u+s \eta^{\prime} \cdot v\right)}\right. & \\
& \left.\times\left.\Omega(u, v) d \sigma(u) d \sigma(v)\right|^{2} \frac{d t d s}{t s}\right)^{1 / 2}
\end{aligned}
$$

is an $L^{\infty}$ function, where $\xi^{\prime}=\xi /|\xi|$ and $\eta^{\prime}=\eta /|\eta|$. It is easy to see that

$$
\begin{aligned}
(m(\xi, \eta))^{2}= & \lim _{M \rightarrow \infty, \varepsilon_{2} \rightarrow 0} \lim _{N \rightarrow \infty, \varepsilon_{1} \rightarrow 0} \int_{\left(\mathbf{S}^{n-1} \times \mathbf{S}^{m-1}\right)^{2}} \Omega(u, v) \overline{\Omega(x, y)} \\
& \times \int_{\varepsilon_{2}}^{M}\left(e^{-2 \pi i s \eta^{\prime} \cdot(v-y)} \frac{d s}{s}\right) \\
& \times \int_{\varepsilon_{1}}^{N}\left(e^{-2 \pi i t \xi^{\prime} \cdot(u-x)} \frac{d t}{t}\right) d \sigma(u) d \sigma(v) d \sigma(x) d \sigma(y) .
\end{aligned}
$$

Notice that

$$
\begin{aligned}
\int_{\varepsilon_{1}}^{N}\left(e^{-2 \pi i t \xi^{\prime} \cdot(u-x)}-\cos (2 \pi t)\right) \frac{d t}{t} & \longrightarrow \\
& \log \left|\xi^{\prime} \cdot(u-x)\right|^{-1}-i \frac{\pi}{2} \operatorname{sgn}\left(\xi^{\prime} \cdot(u-x)\right)
\end{aligned}
$$

as $N \rightarrow \infty$ and $\varepsilon_{1} \rightarrow 0$, and the integral is bounded uniformly in $\varepsilon_{1}$ and $N, C\left(1+|\log | \xi^{\prime} \cdot(u-x)||\right)$. Now, if we choose $\Omega$ to be a real-valued function, by the cancelation conditions on $\Omega$ and invoking Lebesgue 
dominated convergence theorem, we obtain

$$
\begin{aligned}
(m(\xi, \eta))^{2}= & \int_{\left(\mathbf{S}^{n-1} \times \mathbf{S}^{m-1}\right)^{2}}\left(\Omega(u, v) \Omega(x, y) \log \left|\xi^{\prime} \cdot(u-x)\right|^{-1}\right. \\
& \times \log \left|\eta^{\prime} \cdot(v-y)\right|^{-1}-\left(\frac{\pi^{2}}{4} \operatorname{sgn}\left(\xi^{\prime} \cdot(u-x)\right)\right. \\
& \left.\left.\quad \times \operatorname{sgn}\left(\eta^{\prime} \cdot(v-y)\right)\right)\right) d \sigma(u) d \sigma(v) d \sigma(x) d \sigma(y) .
\end{aligned}
$$

For simplicity, we shall construct the function $\Omega$ only in the case $n=m=2$, and we shall work on $[-1,1]^{2}$ instead of $\mathbf{S}^{1} \times \mathbf{S}^{1}$. By (2.1), we notice that Theorem 1.1 (b) is proved if we can construct an $\Omega$ on $[-1,1]^{2}$ with the following properties:

$$
\begin{array}{r}
\int_{[-1,1]^{2}}|\Omega(u, v)|(\log (2+|\Omega(u, v)|))^{1-\varepsilon} d u d v<\infty \\
\text { for each } \varepsilon>0
\end{array}
$$

$$
\begin{aligned}
\mathcal{I}(1,1)=\int_{[0,1]^{2}} \int_{[0,1]^{2}} \Omega(u, v) & \Omega(x, y) \\
& \times F(u, v, x, y) d u d v d x d y=\infty
\end{aligned}
$$

$$
\begin{aligned}
\mathcal{I}(1,2)=\int_{[-1,1]^{2} \backslash[0,1]^{2}} \int_{[0,1]^{2}} \mid & \Omega(u, v) \Omega(x, y) \\
& \times F(u, v, x, y) \mid d u d v d x d y<\infty \\
\mathcal{I}(2,1)=\int_{[0,1]^{2}} \int_{[-1,1]^{2} \backslash[0,1]^{2}} & \mid \Omega(u, v) \Omega(x, y) \\
& \times F(u, v, x, y) \mid d u d v d x d y<\infty
\end{aligned}
$$




$$
\begin{aligned}
& \mathcal{I}(2,2)=\int_{[-1,1]^{2} \backslash[0,1]^{2}} \int_{[-1,1]^{2} \backslash[0,1]^{2}} \mid \Omega(u, v) \Omega(x, y) \\
& \times F(u, v, x, y) \mid d u d v d x d y<\infty
\end{aligned}
$$

where

$$
F(u, v, x, y)=\left(\log |x-u|^{-1}\right)\left(\log |y-v|^{-1}\right) .
$$

For $k \in \mathbf{N}$, let $I_{k}=[(1 / k+1),(1 / k))$ and

$$
b_{k}=\sum_{j=3}^{\infty} \frac{k}{(j+1)[\log (k+j)]^{3}} .
$$

Now, by definition of $b_{k}$, we have

$$
\begin{aligned}
b_{k} & =\sum_{j=3}^{k} \frac{k}{(j+1)[\log (k+j)]^{3}}+\sum_{j=k+1}^{\infty} \frac{k}{(j+1)[\log (k+j)]^{3}} \\
& \leq \frac{k}{(\log k)^{3}}\left(\sum_{j=3}^{k} \frac{1}{(j+1)}\right)+k\left(\sum_{j=k+1}^{\infty} \frac{1}{(j+1)(\log j)^{3}}\right) \\
& \leq C \frac{k}{(\log k)^{2}} .
\end{aligned}
$$

Define $\Omega$ on $[-1,1]^{2}$ by

$$
\begin{aligned}
\Omega(u, v)= & \sum_{j=3}^{\infty} \sum_{k=3}^{\infty} \frac{j k}{[\log (k+j)]^{3}} \chi_{I_{k} \times I_{j}}(u, v)-\chi_{[-1,0]}(v)\left(\sum_{k=3}^{\infty} b_{k} \chi_{I_{k}}(u)\right) \\
& -\chi_{[-1,0]}(u)\left(\sum_{k=3}^{\infty} b_{k} \chi_{I_{k}}(v)\right)+\chi_{[-1,0]^{2}}(u, v)\left(\sum_{k=3}^{\infty} \frac{b_{k}}{k(k+1)}\right)
\end{aligned}
$$

where $\chi_{A}$ represents the characteristic function of a set $A$.

Let us now turn to the proof of (2.2)-(2.8). First, the proof of (2.2) is straightforward. To prove (2.3), it suffices to show that

$$
\int_{[0,1]^{2}}|\Omega(u, v)|(\log (2+|\Omega(u, v)|)) d u d v=\infty .
$$


To see this, notice that

$$
\begin{aligned}
\int_{[0,1]^{2}} \mid \Omega & (u, v) \mid(\log (2+|\Omega(u, v)|)) d u d v \\
& =\sum_{j=3}^{\infty} \sum_{k=3}^{\infty} \frac{j k}{[\log (k+j)]^{3}} \int_{I_{k} \times I_{j}}(\log (2+|\Omega(u, v)|)) d u d v \\
& \geq C \sum_{j=3}^{\infty} \sum_{k=3}^{\infty} \frac{(\log k+\log j)}{j k[\log (k+j)]^{3}} \\
& \geq C \sum_{k=3}^{\infty} \sum_{j=k}^{\infty} \frac{(\log k+\log j)}{j k[\log (k+j)]^{3}} \\
& \geq C \sum_{k=3}^{\infty} \frac{1}{k \log k}=\infty .
\end{aligned}
$$

We now prove (2.4). We divide the integral over $[-1,1]^{2}$ into four parts: over $[0,1]^{2},[-1,0] \times[0,1],[0,1] \times[-1,0]$ and $[-1,0] \times[-1,0]$. By similar calculations as those in the proof of $(2.9)$, we obtain the finiteness of the integral over $[0,1]^{2}$. On the other hand, by definition of $\Omega$, we can see that the integral over $[-1,0] \times[0,1]$ equals to

$$
\sum_{k=3}^{\infty} \frac{b_{k}\left(\log \left(2+b_{k}\right)\right)^{1-\varepsilon}}{k(k+1)}<\infty .
$$

Similarly, we can show that the integral over $[0,1] \times[-1,0]$ is finite. Finally, since

$$
\left(\sum_{k=3}^{\infty} \frac{b_{k}}{k(k+1)}\right) \chi_{[-1,0]} \in L^{\infty},
$$

we have that the integral over $[-1,0] \times[-1,0]$ is finite.

Now, we verify (2.5). Let us first prove $\mathcal{I}(1,1)=\infty$. By definition of $\mathcal{I}(1,1)$, we have

$$
\begin{aligned}
& \mathcal{I}(1,1) \\
& \quad=\sum_{j=3}^{\infty} \sum_{k=3}^{\infty} \sum_{s=3}^{\infty} \sum_{l=3}^{\infty} a_{k, j} a_{l, s} \int_{I_{k} \times I_{j}} \int_{I_{l} \times I_{s}} F(u, v, x, y) d x d y d u d v
\end{aligned}
$$


where

$$
a_{k, j}=\frac{j k}{[\log (k+j)]^{3}} .
$$

Notice that, for each $(u, v) \in I_{k} \times I_{j}$ and $(x, y) \in I_{l} \times I_{s}, F(u, v, x, y) \geq$ 0 . Thus,

$$
\begin{aligned}
\mathcal{I}(1,1) \geq \sum_{j=3}^{\infty} \sum_{k=3}^{\infty} \sum_{s \geq 2(j+1)}^{\infty} & \sum_{l \geq 2(k+1)}^{\infty} a_{k, j} a_{l, s} \\
& \times \int_{I_{k} \times I_{j}} \int_{I_{l} \times I_{s}} F(u, v, x, y) d x d y d u d v
\end{aligned}
$$

Now, for $(u, x) \in I_{k} \times I_{l}$ with $l \geq 2(k+1)$, we have $u \geq 2 x$ and hence $\log |x-u|^{-1} \geq \log k$. Similarly, $\log |y-v|^{-1} \geq \log j$ for $(v, y) \in I_{j} \times I_{s}$ with $s \geq 2(j+1)$. Therefore,

$$
\begin{aligned}
\mathcal{I}(1,1) & \geq C \sum_{j=3}^{\infty} \sum_{s \geq 2(j+1)}^{\infty} \sum_{k=3}^{\infty} \sum_{l \geq 2(k+1)}^{\infty} \frac{\log k \log j}{l k j s[\log (k+j)]^{3}[\log (l+s)]^{3}} \\
& \geq C \sum_{k=3}^{\infty} \sum_{j=3}^{\infty} \sum_{s \geq 2(j+1)}^{\infty} \frac{\log k \log j}{k j s[\log (k+j)]^{3}[\log (k+s)]^{2}} \\
& \geq C \sum_{k=3}^{\infty} \sum_{j=3}^{\infty} \frac{\log k \log j}{k j[\log (k+j)]^{4}} \\
& \geq C \sum_{k=3}^{\infty} \sum_{j \geq k}^{\infty} \frac{\log k \log j}{k j[\log (k+j)]^{4}} \\
& \geq C \sum_{k=3}^{\infty} \frac{\log k}{k}\left(\sum_{j \geq k}^{\infty} \frac{1}{j(\log j)^{3}}\right) \\
& \geq C \sum_{k=3}^{\infty} \frac{1}{k \log k}=\infty .
\end{aligned}
$$

Next, we turn to the proof of $(2.6)$. Divide $[-1,1]^{2} \backslash[0,1]^{2}$ into three parts: $[-1,0] \times[0,1],[0,1] \times[-1,0]$ and $[-1,0] \times[-1,0]$. We notice that the integral over $[-1,0] \times[0,1] \times[0,1]^{2}$ is dominated from above 
by

$$
S=\sum_{k=3}^{\infty} \sum_{j=3}^{\infty} \sum_{s=3}^{\infty} a_{k, j} b_{s}|\mathcal{I}(k)| \mathcal{J}(j, s),
$$

where

$$
\mathcal{J}(j, s)=\int_{I_{j} \times I_{s}} \log |y-v|^{-1} d v d y
$$

and

$$
\mathcal{I}(k)=\int_{I_{k}} \int_{-1}^{0} \log |x-u|^{-1} d x d u .
$$

By elementary calculations, it is easy to verify that the following inequalities hold for some positive constant $C$ independent of $k$ and $j$ :

$$
\begin{aligned}
&|\mathcal{I}(j)| \leq C \frac{1}{j^{2}} ; \\
& \mathcal{J}(j, s) \leq C \frac{\log j}{j^{2} s^{2}} \quad \text { if } \quad s>2 j ; \\
& \mathcal{J}(j, s) \leq C \frac{\log s}{j^{2} s^{2}} \quad \text { if } \quad j>2 s ; \\
& \mathcal{J}(j, s) \leq C \frac{\log s}{s^{4}} \quad \text { if } \quad j / 2 \leq s \leq 2 j .
\end{aligned}
$$

In view of (2.10)-(2.11), we have

$$
S \leq S_{1}+S_{2}+S_{3}
$$

where

$$
\begin{aligned}
& S_{1}=\sum_{k=3}^{\infty} \sum_{j=3}^{\infty} \sum_{s>2 j} \frac{j s}{k[\log (k+j)]^{3}(\log s)^{2}} \mathcal{J}(j, s), \\
& S_{2}=\sum_{k=3}^{\infty} \sum_{s=3}^{\infty} \sum_{j>2 s}^{\infty} \frac{j s}{k[\log (k+j)]^{3}(\log s)^{2}} \mathcal{J}(j, s) \\
& S_{3}=\sum_{k=3}^{\infty} \sum_{j=3}^{\infty} \sum_{j / 2 \leq s \leq 2 j} \frac{j s}{k[\log (k+j)]^{3}(\log s)^{2}} \mathcal{J}(j, s) .
\end{aligned}
$$


By (2.12), we have

$$
\begin{aligned}
S_{1} & \leq C \sum_{k=3}^{\infty} \sum_{j=3}^{\infty} \frac{\log j}{k j[\log (k+j)]^{3}} \sum_{s>2 j} \frac{1}{s(\log s)^{2}} \\
& \leq C \sum_{k=3}^{\infty} \sum_{j=3}^{\infty} \frac{1}{k j[\log (k+j)]^{3}} \\
& \leq C\left(\sum_{k=3}^{\infty} \frac{1}{k[\log (k+3)]^{3 / 2}}\right)\left(\sum_{j=3}^{\infty} \frac{1}{j[\log (3+j)]^{3 / 2}}\right)<\infty
\end{aligned}
$$

The proof of $S_{2}<\infty$ follows by (2.13) and the same argument as proving $S_{1}<\infty$. To prove the finiteness of $S_{3}$, we invoke (2.14) to get

$$
\begin{aligned}
S_{3} & \leq C \sum_{k=3}^{\infty} \sum_{j=3}^{\infty} \frac{j}{k[\log (k+j)]^{3}}\left(\sum_{j / 2 \leq s \leq 2 j} \frac{1}{s^{3} \log s}\right) \\
& \leq C \sum_{k=3}^{\infty} \sum_{j=3}^{\infty} \frac{1}{k j[\log (k+j)]^{3} \log j} \\
& \leq C\left(\sum_{k=3}^{\infty} \frac{1}{k(\log k)^{2}}\right)\left(\sum_{j=3}^{\infty} \frac{1}{j(\log j)^{2}}\right)<\infty .
\end{aligned}
$$

Thus, the integral over $[-1,0] \times[0,1] \times[0,1]^{2}$ is finite. Similarly, the integral over $[0,1] \times[-1,0] \times[0,1]^{2}$ is finite. Also, the integral over $[-1,0] \times[-1,0] \times[0,1]^{2}$ is bounded from above by

$$
\begin{aligned}
C \sum_{k=3}^{\infty} & \sum_{j=3}^{\infty} a_{k, j}|\mathcal{I}(k) \mathcal{I}(j)| \\
& \leq C \sum_{k=3}^{\infty} \sum_{j=3}^{\infty} \frac{1}{k j[\log (k+j)]^{3}} \\
& \leq C\left(\sum_{k=3}^{\infty} \frac{1}{k[\log (k+3)]^{3 / 2}}\right)\left(\sum_{j=3}^{\infty} \frac{1}{j[\log (3+j)]^{3 / 2}}\right)<\infty
\end{aligned}
$$

which ends the proof of (2.6). By following a similar argument as proving (2.6), we obtain $\mathcal{I}(2,1)<\infty$. Now, it remains to verify $(2.8)$. 
Divide $[-1,1]^{2} \backslash[0,1]^{2}$ into three parts: $[-1,0] \times[0,1],[0,1] \times[-1,0]$ and $[-1,0] \times[-1,0]$. As above, we shall only present the proof of the finiteness of the integral over $[-1,0] \times[0,1] \times[-1,0] \times[0,1]$ and over $[-1,0] \times[0,1] \times[0,1] \times[-1,0]$ because the proof of the other cases are similar. We start now by proving the finiteness of the integral over $[-1,0] \times[0,1] \times[-1,0] \times[0,1]$. Notice that the last integral is bounded from above by

$$
\begin{aligned}
& C \sum_{k=3}^{\infty} \sum_{l=3}^{\infty} \frac{k l}{(\log k)^{2}(\log l)^{2}} \mathcal{J}(k, l)\left(\int_{-1}^{0} \int_{-1}^{0} \log |y-v|^{-1} d v d y\right) \\
& \leq C \sum_{k=3}^{\infty} \sum_{l=3}^{\infty} \frac{k l}{(\log k)^{2}(\log l)^{2}} \mathcal{J}(k, l)=S^{*}
\end{aligned}
$$

As above, split $S^{*}$ as

$$
S^{*}=S_{1}^{*}+S_{2}^{*}+S_{3}^{*},
$$

where

$$
\begin{aligned}
S_{1}^{*} & =\sum_{k=3}^{\infty} \sum_{l>2 k}^{\infty} \frac{k l}{(\log k)^{2}(\log l)^{2}} \mathcal{J}(k, l) ; \\
S_{2}^{*} & =\sum_{l=3}^{\infty} \sum_{k>2 l}^{\infty} \frac{k l}{(\log k)^{2}(\log l)^{2}} \mathcal{J}(k, l) ; \\
S_{3}^{*} & =\sum_{k=3}^{\infty} \sum_{k / 2 \leq l \leq 2 k} \frac{k l}{(\log k)^{2}(\log l)^{2}} \mathcal{J}(k, l) .
\end{aligned}
$$

By (2.12), we have

$$
\begin{aligned}
S_{1}^{*} & \leq C \sum_{k=3}^{\infty} \frac{1}{k(\log k)}\left(\sum_{l>2 k}^{\infty} \frac{1}{l(\log l)^{2}}\right) \\
& \leq C \sum_{k=3}^{\infty} \frac{1}{k(\log k)^{2}}<\infty .
\end{aligned}
$$

Similarly, by (2.13) $S_{2}^{*}<\infty$. By (2.14),

$$
\begin{aligned}
S_{3}^{*} & \leq C \sum_{k=3}^{\infty} \frac{k}{(\log k)^{2}} \sum_{k / 2 \leq l \leq 2 k} \frac{1}{l^{3}(\log l)} \\
& \leq C \sum_{k=3}^{\infty} \frac{k}{k(\log k)^{3}}<\infty .
\end{aligned}
$$


This finishes the proof of the finiteness of the integral over $[-1,0] \times$ $[0,1] \times[-1,0] \times[0,1]$. Now, we turn to the proof of the finiteness of the integral over $[-1,0] \times[0,1] \times[0,1] \times[-1,0]$. We notice that the integral over $[-1,0] \times[0,1] \times[0,1] \times[-1,0]$ is bounded from above by

$$
\begin{aligned}
& C \sum_{k=3}^{\infty} \sum_{l=3}^{\infty} \frac{k l}{(\log k)^{2}(\log l)^{2}}|\mathcal{I}(k) \mathcal{I}(j)| \\
& \leq C \sum_{k=3}^{\infty} \sum_{j=3}^{\infty} \frac{1}{k j(\log k)^{2}(\log j)^{2}}<\infty .
\end{aligned}
$$

This completes the proof of Theorem 1.1 (b). $\quad \square$

\section{Some lemmas.}

Lemma 3.1. Let $\mu \in \mathbf{N} \cup\{0\}, a_{\mu}=2^{(\mu+1)}$ and $\Omega_{\mu}(\cdot, \cdot)$ be a function on $\mathbf{S}^{n-1} \times \mathbf{S}^{m-1}$ satisfying the conditions:

(i) $\left\|\Omega_{\mu}\right\|_{L^{2}\left(\mathbf{S}^{n-1} \times \mathbf{S}^{m-1}\right)} \leq a_{\mu}^{2}$,

(ii) $\left\|\Omega_{\mu}\right\|_{L^{1}\left(\mathbf{S}^{n-1} \times \mathbf{S}^{m-1}\right)} \leq 1$, and

(iii) $\Omega_{\mu}$ satisfies the cancelation conditions in (1.3) with $\Omega$ replaced by $\Omega_{\mu}$. Assume that $\Phi, \Psi$ are in $C^{2}([0, \infty))$, convex, and increasing functions with $\Phi(0)=\Psi(0)=0$. Let

$$
\begin{aligned}
I_{\mu, k, j}(\xi, \eta)=\left(\int_{\left[a_{\mu}^{k}, a_{\mu}^{k+1}\right) \times\left[a_{\mu}^{j}, a_{\mu}^{j+1}\right)} \mid \int_{\mathbf{S}^{n-1} \times \mathbf{S}^{m-1}} \Omega_{\mu}(x, y)\right. \\
\left.\quad \times\left. e^{-i(\Phi(t)\langle\xi, x\rangle+\Psi(s)\langle\eta, y\rangle)} d \sigma(x) d \sigma(y)\right|^{2} \frac{d t d s}{t s}\right)^{1 / 2} .
\end{aligned}
$$

Then there exist positive constants $C$ and $\alpha$ such that

(3.1) $\left|I_{\mu, k, j}(\xi, \eta)\right| \leq C(\mu+1)$;

$$
\begin{aligned}
& \left|I_{\mu, k, j}(\xi, \eta)\right| \\
& \quad \leq C(\mu+1)\left(\Phi\left(a_{\mu}^{k+1}\right)|\xi|\right)^{\alpha /(\mu+1)}\left(\Psi\left(a_{\mu}^{j+1}\right)|\eta|\right)^{\alpha /(\mu+1)}
\end{aligned}
$$




$$
\begin{aligned}
& \left|I_{\mu, k, j}(\xi, \eta)\right| \\
& \quad \leq C(\mu+1)\left(\Phi\left(a_{\mu}^{k}\right)|\xi|\right)^{-\alpha /(\mu+1)}\left(\Psi\left(a_{\mu}^{j}\right)|\eta|\right)^{-\alpha /(\mu+1)}
\end{aligned}
$$

(3.4) $\left|I_{\mu, k, j}(\xi, \eta)\right|$

$$
\leq C(\mu+1)\left(\Phi\left(a_{\mu}^{k+1}\right)|\xi|\right)^{\alpha /(\mu+1)}\left(\Psi\left(a_{\mu}^{j}\right)|\eta|\right)^{-\alpha /(\mu+1)} ;
$$

$$
\begin{aligned}
& \left|I_{\mu, k, j}(\xi, \eta)\right| \\
& \quad \leq C(\mu+1)\left(\Phi\left(a_{\mu}^{k}\right)|\xi|\right)^{-\alpha /(\mu+1)}\left(\Psi\left(a_{\mu}^{j+1}\right)|\eta|\right)^{\alpha /(\mu+1)},
\end{aligned}
$$

where $C$ is a constant independent of $k, j, \xi, \eta$ and $\mu$.

Proof. First, by condition (ii) on $\Omega_{\mu}$ it is easy to see that (3.1) holds. Next, by the cancelation properties of $\Omega_{\mu}$ and by a simple change of variables we have

$$
\begin{aligned}
\left|I_{\mu, k, j}(\xi, \eta)\right|^{2} \leq \int_{\left[1, a_{\mu}\right) \times\left[1, a_{\mu}\right)} & \left(\int_{\mathbf{S}^{n-1} \times \mathbf{S}^{m-1}}\left|\Omega_{\mu}(x, y)\right|\right. \\
& \left.\times\left|e^{-i \Phi\left(a_{\mu}^{k} t\right)\langle\xi, x\rangle}-1\right| d \sigma(x) d \sigma(y)\right)^{2} \frac{d t d s}{t s} .
\end{aligned}
$$

Since $\Phi$ is increasing we get

$$
\left|I_{\mu, k, j}(\xi, \eta)\right| \leq C(\mu+1)\left|\Phi\left(a_{\mu}^{k+1}\right) \xi\right| .
$$

Similarly,

$$
\left|I_{\mu, k, j}(\xi, \eta)\right| \leq C(\mu+1)\left|\Psi\left(a_{\mu}^{j+1}\right) \eta\right| .
$$

Now, by Schwarz's inequality we have

$$
\begin{aligned}
\mid \int_{\mathbf{S}^{n-1} \times \mathbf{S}^{m-1}} & \left.\Omega_{\mu}(x, y) e^{-i(\Phi(t)<\xi, x>+\Psi(s)\langle\eta, y\rangle)} d \sigma(x) d \sigma(y)\right|^{2} \\
\leq & \int_{\mathbf{S}^{m-1}}\left|\int_{\mathbf{S}^{n-1}} \Omega_{\mu}(x, y) e^{-i \Phi\left(a_{\mu}^{k} t\right)\langle\xi, x\rangle} d \sigma(x)\right|^{2} d \sigma(y) \\
= & \int_{\mathbf{S}^{m-1}}\left(\int_{\mathbf{S}^{n-1} \times \mathbf{S}^{n-1}} \Omega_{\mu}(x, y) \overline{\Omega_{\mu}(u, y)}\right. \\
& \left.\times e^{-i \Phi\left(a_{\mu}^{k} t\right)\langle\xi, x-u\rangle} d \sigma(x) d \sigma(u)\right) d \sigma(y) .
\end{aligned}
$$


Therefore,

$$
\begin{aligned}
\left|I_{\mu, k, j}(\xi, \eta)\right|^{2} \leq \int_{\mathbf{S}^{m-1}}( & \int_{\mathbf{S}^{n-1} \times \mathbf{S}^{n-1}} \Omega_{\mu}(x, y) \overline{\Omega_{\mu}(u, y)} \\
& \left.\times J_{\mu, k}(\xi, x, u) d \sigma(x) d \sigma(u)\right) d \sigma(y),
\end{aligned}
$$

where

$$
J_{\mu, k}(\xi, x, u)=\int_{1}^{a_{\mu}} e^{-i \Phi\left(a_{\mu}^{k} t\right)\langle\xi, x-u\rangle} \frac{d t}{t} .
$$

We now show that

$$
\left|J_{\mu, k}(\xi, x, u)\right| \leq C(\mu+1)\left|\Phi\left(a_{\mu}^{k}\right) \xi\right|^{-1 / 4}\left|\left\langle\xi^{\prime}, x-u\right\rangle\right|^{-1 / 4}
$$

for some positive constant $C$ independent of $\mu$.

The proof of (3.9) follows by a simple application of van der Corput's lemma. In fact, we notice first that

$$
J_{\mu, k}(\xi, x, u)=\int_{1}^{a_{\mu}} H^{\prime}(t) \frac{d t}{t},
$$

where

$$
H(t)=\int_{1}^{t} e^{-i \Phi\left(a_{\mu}^{k} w\right)\langle\xi, x-u\rangle} d w, \quad 1 \leq t \leq a_{\mu} .
$$

By the assumptions on $\Phi$ and the mean value theorem, we have

$$
\begin{gathered}
\frac{d}{d w}\left(\Phi\left(a_{\mu}^{k} w\right)\right)=a_{\mu}^{k} \Phi^{\prime}\left(a_{\mu}^{k} w\right) \geq \frac{\Phi\left(a_{\mu}^{k} w\right)}{w} \geq \frac{\Phi\left(a_{\mu}^{k}\right)}{t} \\
\text { for } 1 \leq w \leq t \leq a_{\mu} .
\end{gathered}
$$

Thus, by van der Corput's lemma,

$$
|H(t)| \leq\left|\Phi\left(a_{\mu}^{k}\right) \xi\right|^{-1}\left|\left\langle\xi^{\prime}, x-u\right\rangle\right|^{-1} t,
$$

for $1 \leq t \leq a_{\mu}$. Hence by integration by parts,

$$
\left|J_{\mu, k}(\xi, x, u)\right| \leq C(\mu+1)\left|\Phi\left(a_{\mu}^{k}\right) \xi\right|^{-1}\left|\left\langle\xi^{\prime}, x-u\right\rangle\right|^{-1} .
$$


By combining this estimate with the trivial estimate,

$$
\left|J_{\mu, k}(\xi, x, u)\right| \leq(\ln 2)(\mu+1),
$$

we get (3.9). By Schwarz's inequality, condition (i) on $\Omega_{\mu}$ and (3.8)-(3.9), we get

$$
\begin{aligned}
\left|I_{\mu, k, j}(\xi, \eta)\right|^{2} \leq & C(\mu+1)^{2} a_{\mu}^{4}\left|\Phi\left(a_{\mu}^{k}\right) \xi\right|^{-1 / 4} \\
& \times\left(\int_{\mathbf{S}^{n-1} \times \mathbf{S}^{n-1}}\left|\left\langle\xi^{\prime}, x-u\right\rangle\right|^{-1 / 2} d \sigma(x) d \sigma(u)\right)^{1 / 2} .
\end{aligned}
$$

Since the last integral is finite, we get

$$
\left|I_{\mu, k, j}(\xi, \eta)\right| \leq C(\mu+1) a_{\mu}^{2}\left|\Phi\left(a_{\mu}^{k}\right) \xi\right|^{-1 / 8} .
$$

Similarly,

$$
\left|I_{\mu, k, j}(\xi, \eta)\right| \leq C(\mu+1) a_{\mu}^{2}\left|\Psi\left(a_{\mu}^{j}\right) \xi\right|^{-1 / 8} .
$$

By (3.1), (3.6)-(3.7) and (3.10)-(3.11) we obtain (3.2)-(3.5). The proof of the lemma is complete.

By the same argument as in $[\mathbf{1 7}$, p. 57], we get the following:

Lemma 3.2. Let $\varphi$ be a nonnegative, decreasing function on $[0, \infty)$ with $\int_{[0, \infty)} \varphi(t) d t=1$. Then

$$
\left|\int_{[0, \infty)} f\left(x-t y^{\prime}\right) \varphi(t) d t\right| \leq M_{y^{\prime}} f(x),
$$

where

$$
M_{y^{\prime}} f(x)=\sup _{R \in \mathbf{R}} \frac{1}{R} \int_{0}^{R}\left|f\left(x-s y^{\prime}\right)\right| d s
$$

is the Hardy-Littlewood maximal function of $f$ in the direction of $y^{\prime}$.

For $\mu \in \mathbf{N} \cup\{0\}$ and $u^{\prime} \in \mathbf{S}^{n-1}$, let $\mathcal{M}_{\Phi, \mu, u^{\prime}}(f)$ denote the maximal function defined by

$$
\mathcal{M}_{\Phi, \mu, u^{\prime}} f(x)=\sup _{k \in \mathbf{Z}}\left|\int_{a_{\mu}^{k}}^{a_{\mu}^{k+1}} f\left(x-\Phi(t) u^{\prime}\right) \frac{d t}{t}\right| .
$$


Lemma 3.3. Assume that $\Phi$ is in $C^{2}([0, \infty))$, convex, and increasing function with $\Phi(0)=0$. Then

$$
\left\|\mathcal{M}_{\Phi, \mu, u^{\prime}}(f)\right\|_{p} \leq C_{p}(\mu+1)\|f\|_{p}
$$

for $1<p \leq \infty$ and $f \in L^{p}$.

Proof. By a change of variable we have

$$
\mathcal{M}_{\Phi, \mu, u^{\prime}} f(x) \leq \sup _{k \in \mathbf{Z}}\left(\int_{\Phi\left(a_{\mu}^{k}\right)}^{\Phi\left(a_{\mu}^{k+1}\right)}\left|f\left(x-t u^{\prime}\right)\right| \frac{d t}{\Phi^{-1}(t) \Phi^{\prime}\left(\Phi^{-1}(t)\right)}\right) .
$$

Without loss of generality, we may assume that $\Phi(t)>0$ for all $t>0$. By Lemma 3.2 and since the function $1 /\left(\Phi^{-1}(t) \Phi^{\prime}\left(\Phi^{-1}(t)\right)\right)$ is nonnegative, decreasing and its integral over $\left[\Phi\left(a_{\mu}^{k}\right), \Phi\left(a_{\mu}^{k+1}\right)\right]$ is equal to $(\ln 2)(\mu+1)$, we obtain

$$
\mathcal{M}_{\Phi, \mu, u^{\prime}} f(x) \leq C(\mu+1) M_{u^{\prime}} f(x),
$$

By the $L^{p}$ boundedness of $M_{u^{\prime}} f$ with bound independent of $u^{\prime}$ we get (3.12) and the proof of the lemma is concluded.

For $\mu \in \mathbf{N} \cup\{0\}$, let

$E_{k, j, \mu}=\left\{(u, v) \in \mathbf{R}^{n} \times \mathbf{R}^{m}: a_{\mu}^{k} \leq|u|<a_{\mu}^{k+1}\right.$ and $\left.a_{\mu}^{j} \leq|v|<a_{\mu}^{j+1}\right\}$.

For any $\Omega \in L^{1}\left(\mathbf{S}^{n-1} \times \mathbf{S}^{m-1}\right)$, we define the maximal operator

$$
\lambda_{\Omega, \mu}^{*} f(x, y)=\sup _{k, j \in \mathbf{Z}}\left|\lambda_{k, j, \Omega, \mu} * f(x, y)\right|,
$$

where

$$
\begin{aligned}
\lambda_{k, j, \Omega, \mu} & * f(x, y) \\
& =\int_{E_{k, j, \mu}}\left|f\left(x-\Phi(|u|) u^{\prime}, y-\Psi(|v|) v^{\prime}\right)\right| \frac{\left|\Omega\left(u^{\prime}, v^{\prime}\right)\right|}{|u|^{n}|v|^{m}} d u d v .
\end{aligned}
$$

Lemma 3.4. Let $\Omega \in L^{1}\left(\mathbf{S}^{n-1} \times \mathbf{S}^{m-1}\right)$ and let $\Phi$ and $\Psi$ be in $C^{2}([0, \infty))$, convex and increasing functions with $\Phi(0)=\Psi(0)=0$. Then

$$
\left\|\lambda_{\Omega, \mu}^{*}(f)\right\|_{p} \leq C_{p}(\mu+1)^{2}\|\Omega\|_{L^{1}\left(\mathbf{S}^{n-1} \times \mathbf{S}^{m-1}\right)}\|f\|_{p}
$$


for $1<p \leq \infty$ and $f \in L^{p}$, where $C_{p}$ is independent of $\Omega, \mu$ and $f$.

Proof. Using polar coordinates we get

$$
\begin{aligned}
\left|\lambda_{k, j, \Omega, \mu} * f(x, y)\right| \leq & \int_{\left[a_{\mu}^{k}, a_{\mu}^{k+1}\right) \times\left[a_{\mu}^{j}, a_{\mu}^{j+1}\right)} \int_{\mathbf{S}^{n-1} \times \mathbf{S}^{m-1}}\left|\Omega\left(u^{\prime}, v^{\prime}\right)\right| \\
& \times\left|f\left(x-\Phi(t) u^{\prime}, y-\Psi(s) v^{\prime}\right)\right| d \sigma\left(u^{\prime}\right) d \sigma\left(v^{\prime}\right) \frac{d t d s}{t s} .
\end{aligned}
$$

Therefore,

$$
\begin{aligned}
\lambda_{\Omega, \mu}^{*} f(x, y) \leq C \int_{\mathbf{S}^{n-1} \times \mathbf{S}^{m-1}} \mid & \left|\Omega\left(u^{\prime}, v^{\prime}\right)\right| \\
& \times\left(\mathcal{M}_{\Psi, \mu, v^{\prime}} \circ \mathcal{M}_{\Phi, \mu, u^{\prime}}\right) f(x, y) d \sigma\left(u^{\prime}\right) d \sigma\left(v^{\prime}\right),
\end{aligned}
$$

where "o" denotes the composition of operators. By Lemma 3.3 and noticing that

$$
\begin{aligned}
& \left\|\lambda_{\Omega, \mu}^{*}(f)\right\|_{p} \\
& \quad \leq C \int_{\mathbf{S}^{n-1} \times \mathbf{S}^{m-1}}\left|\Omega\left(u^{\prime}, v^{\prime}\right)\right|\left\|\left(\mathcal{M}_{\Psi, \mu, v^{\prime}} \circ \mathcal{M}_{\Phi, \mu, u^{\prime}}\right)(f)\right\|_{p} d \sigma\left(u^{\prime}\right) d \sigma\left(v^{\prime}\right),
\end{aligned}
$$

we get (3.15) which ends the proof of the lemma.

Let $\mathcal{M}_{S}$ be the spherical maximal operator defined by

$$
\mathcal{M}_{S} f(x)=\sup _{r>0} \int_{\mathbf{S}^{n-1}}|f(x-r \theta)| d \sigma(\theta) .
$$

By applying Stein's and Bourgain's results, see $[\mathbf{1 6}]$ and $[\mathbf{6}]$, we have

Lemma 3.5. Suppose that $n \geq 2$ and $p>n^{\prime}$. Then $\mathcal{M}_{S}(f)$ is bounded on $L^{p}\left(\mathbf{R}^{n}\right)$.

We shall need the spherical maximal operator $\mathcal{M}_{S P}$ defined on functions $f(x, y)$ on $\mathbf{R}^{n} \times \mathbf{R}^{m}$ by

$$
\mathcal{M}_{S P} f(x, y)=\sup _{r, s>0} \int_{\mathbf{S}^{n-1} \times \mathbf{S}^{m-1}}|f(x-r \theta, y-s v)| d \sigma(\theta) d \sigma(v) .
$$


Define the operators $\mathcal{M}_{S}^{(1)}$ and $\mathcal{M}_{S}^{(2)}$ on functions $f$ on $\mathbf{R}^{n} \times \mathbf{R}^{m}$ by $\left.\left(\mathcal{M}_{S}^{(1)} f\right)(x, y)=\left(\mathcal{M}_{S}^{(1)}\right) f(\cdot, y)\right)(x)$ and $\left.\left(\mathcal{M}_{S}^{(2)} f\right)(x, y)=\left(\mathcal{M}_{S}^{(2)}\right) f(x, \cdot)\right)$ $(y)$. By invoking Lemma 3.5 and the inequality

$$
\mathcal{M}_{S P} f(x, y) \leq\left(\mathcal{M}_{S}^{(2)} \circ \mathcal{M}_{S}^{(1)}\right) f(x, y)
$$

we get the following:

Lemma 3.6. Suppose that $n, m \geq 2$ and $p>\max \left\{n^{\prime}, m^{\prime}\right\}$. Then $\mathcal{M}_{S P}(f)$ is bounded on $L^{p}\left(\mathbf{R}^{n} \times \mathbf{R}^{m}\right)$.

4. Proof of Theorem 1.2. We start with proving part (a) of Theorem 1.2. Assume that $\Omega$ satisfies (1.3) and belongs to $L(\log L)^{2 / \gamma^{\prime}}\left(\mathbf{S}^{n-1} \times \mathbf{S}^{m-1}\right)$ for $1 \leq \gamma \leq 2$. Decompose $\Omega$ as in [2], (see also [4]). For $\mu \in \mathbf{N}$, let $\mathbf{E}_{\mu}$ be the set of points $(x, y) \in \mathbf{S}^{n-1} \times \mathbf{S}^{m-1}$ which satisfy $2^{\mu} \leq|\Omega(x, y)|<2^{\mu+1}$. Also, we let $\mathbf{E}_{0}$ be the set of all those points $(x, y) \in \mathbf{S}^{n-1} \times \mathbf{S}^{m-1}$ which satisfy $|\Omega(x, y)|<2$. For $\mu \in$ $\mathbf{N} \cup\{0\}$, set $b_{\mu}=\Omega \chi_{\mathbf{E}_{\mu}}$ and $\omega_{\mu}=\left\|b_{\mu}\right\|_{1}$. Set $I=\left\{\mu \in \mathbf{N}: \omega_{\mu} \geq 2^{-4 \mu}\right\}$ and define the sequence of functions $\left\{\Omega_{\mu}\right\}_{\mu \in I \cup\{0\}}$ by

$$
\begin{aligned}
\Omega_{0}(x, y)= & \sum_{\mu \in\{0\} \cup(\mathbf{N}-I)} b_{\mu}(x, y)-\sum_{\mu \in\{0\} \cup(\mathbf{N}-I)}\left(\int_{\mathbf{S}^{n-1}} b_{\mu}(x, y) d \sigma(x)\right) \\
& -\sum_{\mu \in\{0\} \cup(\mathbf{N}-I)}\left(\int_{\mathbf{S}^{m-1}} b_{\mu}(x, y) d \sigma(y)\right) \\
& +\sum_{\mu \in\{0\} \cup(\mathbf{N}-I)} \int_{\mathbf{S}^{n-1} \times \mathbf{S}^{m-1}} b_{\mu}(x, y) d \sigma(x) d \sigma(y),
\end{aligned}
$$

and for $\mu \in I$,

$$
\begin{array}{r}
\Omega_{\mu}(x, y)=\left(\omega_{\mu}\right)^{-1}\left(b_{\mu}(x, y)-\int_{\mathbf{S}^{n-1}} b_{\mu}(x, y) d \sigma(x)-\int_{\mathbf{S}^{m-1}} b_{\mu}(x, y) d \sigma(y)\right. \\
\left.+\int_{\mathbf{S}^{n-1} \times \mathbf{S}^{m-1}} b_{\mu}(x, y) d \sigma(x) d \sigma(y)\right) .
\end{array}
$$


Then one can easily verify that the following hold for all $\mu \in I \cup\{0\}$ and for some positive constant $C$ :

$$
\begin{gathered}
\left\|\Omega_{\mu}\right\|_{2} \leq C a_{\mu}^{2}, \quad\left\|\Omega_{\mu}\right\|_{1} \leq C ; \\
\sum_{\mu \in I \cup\{0\}}(\mu+1)^{2 / \gamma^{\prime}} \omega_{\mu} \leq C\|\Omega\|_{L(\log L)^{2 / \gamma^{\prime}}\left(\mathbf{S}^{n-1} \times \mathbf{S}^{m-1}\right)} ; \\
\int_{\mathbf{S}^{n-1}} \Omega_{\mu}(u, \cdot) d \sigma(u)=\int_{\mathbf{S}^{m-1}} \Omega_{\mu}(\cdot, v) d \sigma(v)=0 ; \\
\Omega=\sum_{\mu \in I \cup\{0\}} \omega_{\mu} \Omega_{\mu} .
\end{gathered}
$$

By (4.4) we have

$$
\mathcal{S}_{\Omega, \Phi, \Psi} f(x, y) \leq \sum_{\mu \in I \cup\{0\}} \omega_{\mu} \mathcal{S}_{\Omega_{\mu}, \Phi, \Psi} f(x, y) .
$$

By (4.5) it suffices to show that the inequality

$\left\|\mathcal{S}_{\Omega_{\mu}, \Phi, \Psi} f\right\|_{p} \leq C_{p}(\mu+1)^{2 / \gamma^{\prime}}\|f\|_{p} \quad$ for all $\quad \gamma^{\prime} \leq p<\infty \quad$ and $\quad f \in L^{p}$

holds for $\gamma^{\prime} \leq p<\infty$ if $1<\gamma \leq 2$ and for $p=\infty$ if $\gamma=1$. To prove (4.6), we need to consider three cases. We first prove (4.6) for the case $\gamma=2$.

The case $\gamma=2$. Since $\Phi$ is convex and increasing in $(0, \infty), \Phi(t) / t$ is also increasing for $t>0$. Therefore, for $\mu \in \mathbf{N} \cup\{0\}$, the sequence $\left\{\Phi\left(a_{\mu}^{k}\right): k \in \mathbf{Z}\right\}$ is a lacunary sequence with $\Phi\left(a_{\mu}^{k+1}\right) / \Phi\left(a_{\mu}^{k}\right) \geq a_{\mu}>1$. Let $\left\{\psi_{k, \mu, \Phi}\right\}_{-\infty}^{\infty}$ be a smooth partition of unity in $(0, \infty)$ adapted to the interval $E_{k, \mu, \Phi}=\left[\left(\Phi\left(a_{\mu}^{k+1}\right)\right)^{-1},\left(\Phi\left(a_{\mu}^{k-1}\right)\right)^{-1}\right]$. To be precise, we require the following:

$$
\begin{gathered}
\psi_{k, \mu, \Phi} \in C^{\infty}, 0 \leq \psi_{k, \mu, \Phi} \leq 1, \sum_{k} \psi_{k, \mu, \Phi}(t)=1, \\
\operatorname{supp} \psi_{k, \mu, \Phi} \subseteq E_{k, \mu, \Phi},\left|\frac{d^{s} \psi_{k, \mu, \Phi}(t)}{d t^{s}}\right| \leq \frac{C_{s}}{t^{s}},
\end{gathered}
$$


where $C_{s}$ is independent of the lacunary sequence $\left\{\Phi\left(a_{\mu}^{k}\right): k \in \mathbf{Z}\right\}$. Define the multiplier operators $S_{k, j, \mu}$ in $\mathbf{R}^{n} \times \mathbf{R}^{m}$ by

$$
\left(\widehat{S_{k, j, \mu} f}\right)(\xi, \eta)=\psi_{k, \mu, \Phi}(|\xi|) \psi_{j, \mu, \Psi}(|\eta|) \hat{f}(\xi, \eta) .
$$

Then for any $f \in \mathcal{S}\left(\mathbf{R}^{n} \times \mathbf{R}^{m}\right)$ and $l, s \in \mathbf{Z}$ we have

$$
f(x, y)=\sum_{k, j \in \mathbf{Z}}\left(S_{k+l, j+s, \mu} f\right)(x, y) .
$$

By duality we have

$$
\begin{aligned}
\mathcal{S}_{\Omega_{\mu}, \Phi, \Psi}^{(2)} f(x, y) & =\left(\int_{(0, \infty) \times(0, \infty)}\left|F_{r, t, \Omega_{\mu}} f(x, y)\right|^{2} \frac{d r d t}{r t}\right)^{1 / 2} \\
& =\left(\sum_{k, j \in \mathbf{Z}} \int_{\left[a_{\mu}^{k}, a_{\mu}^{k+1}\right) \times\left[a_{\mu}^{j}, a_{\mu}^{j+1}\right)}\left|F_{r, t, \Omega_{\mu}}(x, y)\right|^{2} \frac{d r d t}{r t}\right)^{1 / 2},
\end{aligned}
$$

where

$$
\begin{aligned}
& F_{r, t, \Omega} f(x, y) \\
& \quad=\int_{\mathbf{S}^{n-1} \times \mathbf{S}^{m-1}} f(x-\Phi(r) \xi, y-\Psi(t) \eta) \Omega(\xi, \eta) d \sigma(\xi) d \sigma(\eta) .
\end{aligned}
$$

By Minkowski's inequality it is easy to see that

$$
\begin{aligned}
& \mathcal{S}_{\Omega_{\mu}, \Phi, \Psi}^{(2)} f(x, y) \\
& \leq\left(\sum_{k, j \in \mathbf{Z}} \int_{\left[a_{\mu}^{k}, a_{\mu}^{k+1}\right) \times\left[a_{\mu}^{j}, a_{\mu}^{j+1}\right)}\left|\sum_{l, s \in \mathbf{Z}} H_{k+l, j+s, r, t, \mu, \Omega_{\mu}} f(x, y)\right|^{2} \frac{d r d t}{r t}\right)^{1 / 2} \\
& \leq \sum_{l, s \in \mathbf{Z}}\left(\sum_{k, j \in \mathbf{Z}} \int_{\left[a_{\mu}^{k}, a_{\mu}^{k+1}\right) \times\left[a_{\mu}^{j}, a_{\mu}^{j+1}\right)}\left|H_{k+l, j+s, r, t, \mu, \Omega_{\mu}} f(x, y)\right|^{2} \frac{d r d t}{r t}\right)^{1 / 2}
\end{aligned}
$$

where

$$
\begin{aligned}
& H_{l, s, t, r, \mu, \Omega} f(x, y) \\
& \quad=\int_{\mathbf{S}^{n-1} \times \mathbf{S}^{m-1}} \Omega(\xi, \eta)\left(S_{l, s, \mu} f\right)(x-\Phi(r) \xi, y-\Psi(t) \eta) d \sigma(\xi) d \sigma(\eta) .
\end{aligned}
$$


Now if we let

$$
\begin{aligned}
T_{l, s, \mu, \Omega_{\mu}} & f(x, y) \\
& =\sum_{k, j \in \mathbf{Z}} \int_{\left[a_{\mu}^{k}, a_{\mu}^{k+1}\right) \times\left[a_{\mu}^{j}, a_{\mu}^{j+1}\right)}\left|H_{k+l, j+s, r, t, \mu, \Omega_{\mu}} f(x, y)\right|^{2} \frac{d r d t}{r t},
\end{aligned}
$$

then we have

$$
\mathcal{S}_{\Omega_{\mu}, \Phi, \Psi}^{(2)} f(x, y) \leq \sum_{l, s \in \mathbf{Z}} T_{l, s, \mu, \Omega_{\mu}} f(x, y) .
$$

Therefore, to prove (4.6), it suffices to prove

$$
\left\|T_{l, s, \mu, \Omega_{\mu}}(f)\right\|_{p} \leq C_{p}(\mu+1) 2^{-\theta_{p}|l|} 2^{-\theta_{p}|s|}\|f\|_{p}
$$

for some positive constants $C_{p}, \theta_{p}$ and for all $2 \leq p<\infty$.

The proof of (4.8) follows by interpolation between a sharp $L^{2}$ estimate and a cruder $L^{p}$ estimate of $T_{l, s, \mu, \Omega_{\mu}}(f)$.

First, the $L^{2}$ boundedness of $T_{l, s, \mu, \Omega_{\mu}}(f)$ is provided by a simple application of Plancherel's theorem and using Lemma 3.1.

$$
\begin{aligned}
& \left\|T_{l, s, \mu, \Omega_{\mu}}(f)\right\|_{2}^{2} \\
& =\int_{\mathbf{R}^{n} \times \mathbf{R}^{m}} \sum_{k, j \in \mathbf{Z}} \int_{\left[a_{\mu}^{k}, a_{\mu}^{k+1}\right) \times\left[a_{\mu}^{j}, a_{\mu}^{j+1}\right)}\left|H_{k+l, j+s, r, t, \mu, \Omega_{\mu}} f(x, y)\right|^{2} \frac{d r d t}{r t} d x d y \\
& \leq\left.\sum_{k, j \in \mathbf{Z}} \int_{\Delta_{k+l, j+s}} \int_{\left[a_{\mu}^{k}, a_{\mu}^{k+1}\right) \times\left[a_{\mu}^{j}, a_{\mu}^{j+1}\right)} \Omega_{\mu}(x, y) e^{-i(\Phi(r)\langle\xi, x\rangle+\Psi(t)\langle\eta, y\rangle)} d \sigma(x) d \sigma(y)\right|^{2} \frac{d r d t}{r t} \\
& \qquad \int_{\mathbf{S}^{n-1} \times \mathbf{S}^{m-1}} \\
& \leq\left.\hat{f}(\xi, \eta)\right|^{2} d \xi d \eta \sum_{k, j \in \mathbf{Z}} \int_{\Delta_{k+l, j+s}}|\hat{f}(\xi, \eta)|^{2} d \xi d \eta \\
& \leq C(\mu+1)^{2} 2^{-2 \alpha|l|} 2^{-2 \alpha|s|} \int_{2}, \\
& \leq C(\mu+1)^{2} 2^{-2 \alpha|l|} 2^{-2 \alpha|s|}\|f\|_{2}^{2},
\end{aligned}
$$

where

$$
\Delta_{k, j}=\left\{(\xi, \eta) \in \mathbf{R}^{n} \times \mathbf{R}^{m}:(|\xi|,|\eta|) \in E_{k, \mu, \Phi} \times E_{j, \mu, \Psi}\right\}
$$


Therefore, we have

$$
\left\|T_{l, s, \mu, \Omega_{\mu}}(f)\right\|_{2} \leq C(\mu+1) 2^{-\alpha|l|} 2^{-\alpha|s|}\|f\|_{2} .
$$

On the other hand, we need to compute the $L^{p}$-norm of $T_{l, s, \mu, \Omega_{\mu}}(f)$ for $p>2$. By duality, there is a function $g$ in $L^{(p / 2)^{\prime}}\left(\mathbf{R}^{n} \times \mathbf{R}^{m}\right)$ with $\|g\|_{(p / 2)^{\prime}} \leq 1$ such that

$$
\begin{aligned}
\| & T_{l, s, \mu, \Omega_{\mu}}(f) \|_{p}^{2} \\
= & \sum_{k, j \in \mathbf{Z}} \int_{\mathbf{R}^{n} \times \mathbf{R}^{m}} \int_{\left[a_{\mu}^{k}, a_{\mu}^{k+1}\right) \times\left[a_{\mu}^{j}, a_{\mu}^{j+1}\right)}\left|H_{k+l, j+s, r, t, \mu, \Omega_{\mu}} f(x, y)\right|^{2} \frac{d r d t}{r t} \\
\leq & \left\|\Omega_{\mu}\right\|_{1} \sum_{k, j \in \mathbf{Z}} \int_{\mathbf{R}^{n} \times \mathbf{R}^{m}} \int_{\left[a_{\mu}^{k}, a_{\mu}^{k+1}\right) \times\left[a_{\mu}^{j}, a_{\mu}^{j+1}\right)} \int_{\mathbf{S}^{n-1} \times \mathbf{S}^{m-1}}\left|\Omega_{\mu}(\xi, y)\right| d x d y \\
& \times\left|S_{k+l, j+s, \mu} f(x, y)\right|^{2}|g(x+\Phi(r) \xi, y+\Psi(t) \eta)| d \sigma(\xi) d \sigma(\eta) \frac{d r d t}{r t} d x d y \\
\leq & C \sum_{k, j \in \mathbf{Z}} \int_{\mathbf{R}^{n} \times \mathbf{R}^{m}}\left|S_{k+l, j+s, \mu} f(x, y)\right|^{2} \lambda_{\Omega_{\mu}, \mu}^{*}(\tilde{g})(-x,-y) d x d y \\
\leq & C \sum_{l, s \in \mathbf{Z}}\left|S_{k+l, j+s, \mu} f\right|^{2}\left\|_{p / 2}\right\| \lambda_{\Omega_{\mu}, \mu}^{*}(\tilde{g}) \|_{(p / 2)^{\prime}},
\end{aligned}
$$

where $\tilde{g}(x, y)=g(-x,-y)$.

By (4.1), invoking Lemma 3.4 and using the Littlewood-Paley theory and Theorem 3 along with the remark that follows its statement in $[\mathbf{1 5}$, p. 96], we have

$$
\left\|T_{l, s, \mu, \Omega_{\mu}}(f)\right\|_{p} \leq C_{p}(\mu+1)\|f\|_{p} \quad \text { for } \quad 2 \leq p<\infty .
$$

Now, (4.8) follows by interpolating between (4.9) and (4.10). This completes the proof of (4.6) in the case $\gamma=2$. 
The case $\gamma=1$. If $f \in L^{\infty}\left(\mathbf{R}^{n} \times \mathbf{R}^{m}\right)$ and $h \in L^{1}\left(\mathbf{R}^{+} \times\right.$ $\left.\mathbf{R}^{+}, d s d t /(s t)\right)$, then

$$
\begin{array}{r}
\mid \int_{0}^{\infty} \int_{0}^{\infty} h(t, s) \int_{\mathbf{S}^{n-1} \times \mathbf{S}^{m-1}} f(x-\Phi(t) u, y-\Psi(s) v) \\
\times \Omega_{\mu}(u, v) d \sigma(u) d \sigma(v) \frac{d t d s}{t s} \mid \\
\leq C\|f\|_{L^{\infty}}\|h\|_{L^{1}\left(\mathbf{R}^{+} \times \mathbf{R}^{+}, d s d t /(s t)\right)}
\end{array}
$$

for every $(x, y) \in \mathbf{R}^{n} \times \mathbf{R}^{m}$. By taking the supremum on both sides of the above inequality over all radial functions $h$ with

$$
\|h\|_{L^{1}\left(\mathbf{R}^{+} \times \mathbf{R}^{+}, d s d t /(s t)\right)} \leq 1
$$

yields

$$
\mathcal{S}_{\Omega_{\mu}, \Phi, \Psi}^{(1)} f(x, y) \leq C\|f\|_{L^{\infty}\left(\mathbf{R}^{n} \times \mathbf{R}^{m}\right)}
$$

for almost every $(x, y) \in \mathbf{R}^{n} \times \mathbf{R}^{m}$. Hence,

$$
\left\|\mathcal{S}_{\Omega_{\mu}, \Phi, \Psi}^{(1)} f\right\|_{L^{\infty}\left(\mathbf{R}^{n} \times \mathbf{R}^{m}\right)} \leq C\|f\|_{L^{\infty}\left(\mathbf{R}^{n} \times \mathbf{R}^{m}\right)} .
$$

The case $1<\gamma<2$. We shall use an idea employed in the oneparameter case in $[\mathbf{1 4}]$. By duality,

$$
\begin{aligned}
\mathcal{S}_{\Omega_{\mu}, \Phi, \Psi}^{(\gamma)} f(x, y)= & \| \int_{\mathbf{S}^{n-1} \times \mathbf{S}^{m-1}} f(x-\Phi(t) u, y \\
& -\Psi(s) v) \Omega_{\mu}(u, v) d \sigma(u) d \sigma(v) \|_{L^{\gamma^{\prime}}\left(\mathbf{R}^{+} \times \mathbf{R}^{+}, d s d t /(s t)\right)} .
\end{aligned}
$$

Thus,

$$
\left\|\mathcal{S}_{\Omega_{\mu}, \Phi, \Psi}^{(\gamma)} f\right\|_{L^{p}\left(\mathbf{R}^{n} \times \mathbf{R}^{m}\right)}=\|S(f)\|_{L^{p}\left(L^{\gamma^{\prime}}\left(\mathbf{R}^{+} \times \mathbf{R}^{+}, d s d t /(s t)\right), \mathbf{R}^{n} \times \mathbf{R}^{m}\right)}
$$

where

$$
S: L^{p}\left(\mathbf{R}^{n} \times \mathbf{R}^{m}\right) \longrightarrow L^{p}\left(L^{\gamma^{\prime}}\left(\mathbf{R}^{+} \times \mathbf{R}^{+}, d s d t /(s t)\right), \mathbf{R}^{n} \times \mathbf{R}^{m}\right)
$$


defined by

$$
\begin{aligned}
& S(f)(x, y, t, s) \\
& \quad=\int_{\mathbf{S}^{n-1} \times \mathbf{S}^{m-1}} f(x-\Phi(t) u, y-\Psi(s) v) \Omega_{\mu}(u, v) d \sigma(u) d \sigma(v) .
\end{aligned}
$$

By (4.6), for $\gamma=2$, and (4.11), we interpret that

$$
\begin{gathered}
\|S(f)\|_{L^{p}\left(L^{2}\left(\mathbf{R}^{+} \times \mathbf{R}^{+}, d s d t /(s t)\right), \mathbf{R}^{n} \times \mathbf{R}^{m}\right)} \leq C(\mu+1)\|f\|_{L^{p}\left(\mathbf{R}^{n} \times \mathbf{R}^{m}\right)} \\
\text { for } \quad 2 \leq p<\infty
\end{gathered}
$$

and

$$
\|S(f)\|_{L^{\infty}\left(L^{\infty}\left(\mathbf{R}^{+} \times \mathbf{R}^{+}, d s d t /(s t)\right), \mathbf{R}^{n} \times \mathbf{R}^{m}\right)} \leq C\|f\|_{L^{\infty}\left(\mathbf{R}^{n} \times \mathbf{R}^{m}\right)} .
$$

Applying the real interpolation theorem for Lebesgue mixed normed spaces to the above results, see [5], we conclude that

$$
\begin{gathered}
\|S(f)\|_{L^{p}\left(L^{\gamma^{\prime}}\left(\mathbf{R}^{+} \times \mathbf{R}^{+}, d s d t /(s t)\right), \mathbf{R}^{n} \times \mathbf{R}^{m}\right)} \leq C(\mu+1)^{2 / \gamma^{\prime}}\|f\|_{L^{p}\left(\mathbf{R}^{n} \times \mathbf{R}^{m}\right)} \\
\text { for } \quad \gamma^{\prime} \leq p<\infty,
\end{gathered}
$$

which in turn implies (4.6) for $1<\gamma<2$. The proof of Theorem 1.2 is complete.

A proof of part (b) of Theorem 1.2 can be constructed by the above estimates and following the same argument as in [1]. Details are omitted.

Acknowledgments. The authors wish to thank the referee very much for his helpful comments.

\section{REFERENCES}

1. H. Al-Qassem, $L^{p}$ estimates for a rough maximal operator on product spaces, J. Korean Math. Soc. 42 (2005), 405-434.

2. H. Al-Qassem, A. Al-Salman and Y. Pan, Singular integrals on product domains, Indiana Univ. Math. J., to appear.

3. H. Al-Qassem and Y. Pan, $L^{p}$ boundedness for singular integrals with rough kernels on product domains, Hokkaido Math. J. 31 (2002), 555-613. 
4. A. Al-Salman and Y. Pan, Singular integrals with rough kernels in $L \log ^{+} L$ $\left(\mathbf{S}^{n-1}\right)$, J. London Math. Soc. (2) 66 (2002), 153-174.

5. A. Benedek and R. Panzone, The spaces $L^{p}$, with mixed norm, Duke Math. J. 28 (1961), 301-324.

6. J. Bourgain, Average in the plane over convex curves and maximal operators, J. Analyse Math. 47 (1986), 69-85.

7. L.K. Chen and H. Lin, A maximal operator related to a class of singular integrals, Illinois. J. Math. 34 (1990), 120-126.

8. Y. Ding, A note on a class of rough maximal operators on product domains, J. Math. Anal. Appl. 232 (1999), 222-228.

9. J. Duoandikoetxea, Multiple singular integrals and maximal functions along hypersurfaces, Ann. Inst. Fourier (Grenoble) 36 (1986), 185-206.

10. J. Duoandikoetxea and J.L. Rubio de Francia, Maximal functions and singular integral operators via Fourier transform estimates, Invent. Math. 84 (1986), 541-561.

11. D. Fan and Y. Pan, A singular integral with rough kernel, Proc. Amer. Math. Soc. 125 (1997), 3695-3703.

12. R. Fefferman, Singular integrals on product domains, Bull. Amer. Math. Soc. 4 (1981), 195-201.

13. R. Fefferman and E.M. Stein, Singular integrals on product spaces, Adv. Math. 45 (1982) 117-143.

14. H.V. Le, Maximal operators and singular integral operators along submanifolds, J. Math. Anal. Appl. 296 (2004), 44-64.

15. E.M. Stein, Singular integrals and differentiability properties of functions, Princeton Univ. Press, Princeton, 1970.

16. - Maximal functions: Spherical means, Proc. Nat. Acad. Sci. 73 (1976), $2174-2175$.

17. - Harmonic analysis real-variable methods, orthogonality and oscillatory integrals, Princeton Univ. Press, Princeton, 1993.

Department of Mathematics, Yarmouk University, Irbid-Jordan

E-mail address: husseink@yu.edu.jo

Department of Mathematics, University of Pittsburgh, Pittsburgh, PA 15260

E-mail address: yibiao@pitt.edu 